\title{
RESEARCH
}

Open Access

\section{Reactionary and reparative dentin formation after pulp capping: Hydrogel vs. Dycal}

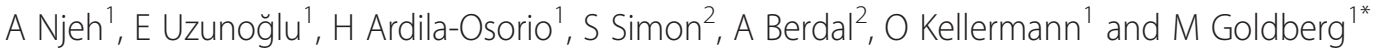

\begin{abstract}
Background: After indirect capping, injured odontoblasts generate reactionary dentin, whereas after direct capping of a pulp exposure pulp, cells stimulate the formation of reparative dentin. The aim of this study was to evaluate and compare the effects of two direct capping agents on pulp tissue reactions: Hydrogel (a bovine serum albumin (BSA)/glutaraldehyde,) and Dycal (a calcium hydroxide-based capping agent).

Methods: In 6-week-old male Sprague-Dawley rats, occlusal cavities were drilled in the first maxillary molars, and the pulps were exposed. In one of the groups, 24 right molars were capped with Hydrogel (G1), whereas in the other group $24 \mathrm{M}$ were capped with Dycal (G2). After 1 to 4 weeks, the rats were anaesthetized intraperitoneally (six rats per group) and perfused intracardiacally with $4 \%$ paraformaldehyde fixative. Maxillary molar's blocks were demineralized with a $4.13 \%$ EDTA solution, embedded in paraffin, and the sections were histologically stained. Measurements of the thickness of reactionary dentin and area of inflammation were measured with ImageJ software. Results were compared with Kruskal Wallis and Mann Whitney $U$ tests at $p=0.05$.
\end{abstract}

Results: One week after Dycal capping, a statistically significant large number of aggregates of pulp cells enlightened pulpal inflammation compared to Hydrogel. At 2-3 weeks, reactionary dentin formation was increased at the periphery of the pulp chamber. After 4 weeks, a dentinal bridge sealed partially the pulp exposure, while tunnel defects persisting across reparative osteodentin. In contrast, 1 week after Hydrogel capping, inflammation was barely detectable. Hydrogel induced the massive apposition of reactionary dentin at the pulp periphery, and reparative dentin was developing within the pulp. The degradation of Hydrogel releases glutaraldehyde acting on pulp cells as a fixative and consequently favoring BSA bioactivity.

Conclusion: After Hydrogel capping, nemosis stimulates pulp mineralization, improving reactionary and reparative dentin formation. In contrast, the highly alkaline compound Dycal produced inflammation within the pulp. The differences between the two capping agents suggest that Hydrogel might present some clinical advantages over Dycal.

Keywords: Biomaterial(s), Inflammation, Mineralized tissue/development, Pulp biology, Pulp capping, Restorative dentistry

\footnotetext{
* Correspondence: mgoldod@gmail.com

${ }^{1}$ Faculté des Sciences Fondamentales et Biomédicales, INSERM UMR-S 1124 and Université Sorbonne Paris Cité, 45 rue des Saints Pères, 75270 Cedex 06 Paris, France

Full list of author information is available at the end of the article
} 


\section{Background}

In dental tissues, two types of pathological dentin have been identified after the adverse effects due to either slow dental caries or rapid pulp exposure. In cariousexposed teeth, odontoblasts and cells from the subodontoblastic Hoehl's layer were implicated in the formation of reactionary dentin. Beneath calciotraumatic lines, indirect capping induced the formation of bonelike (osteo) dentin. Deeper lesions combined with pulp exposure contributed to the construction of reparative dentin by pulp cells. Indirect capping produced reactionary dentin, while direct pulp capping led to the formation of reparative dentin by a dentinal bridge, closing almost the whole pulp exposure.

Since the pioneering work of Hermann (Hermann 1930), calcium hydroxide $\left[\mathrm{Ca}(\mathrm{OH})_{2}\right]$ is recognized as a beneficial pulp capping agent in dentistry. The high alkaline $\mathrm{pH}$ causes a controlled wound and subsequently a scar at the surface of the exposed pulp. Reparative cells are recruited in the central part of the pulp. The high $\mathrm{pH}(\mathrm{pH} 12)$ constitutes an important parameter for the biological properties of $\mathrm{Ca}(\mathrm{OH})_{2}$. Inflammatory cells migrate towards the wounded area. At the border located between the necrotic and vital tissues, treatment with Dycal induces the necrosis of a limited area. Adjacent to the necrotic zone, a reparative dentinal bridge starts to be formed (Tronstad 1974).

The initial reaction of $\mathrm{Ca}(\mathrm{OH})_{2}$ on the dental pulp is vascular and associated with cell migration, proliferation and liquefaction necrosis (necrotic pulp) (Schroder 1985). These conditions are required to promote the biomaterial mineralization and cellular differentiation. Matrix vesicles initiate the formation and diffusion of mineralized granules in the newly formed collagen layer. Secondary odontoblasts differentiate, including the socalled Hoehl's cells and mesenchymal stem cells/pulp progenitors. After capping, crystallite structures are observed at the interface between the superficial necrotic zone and the underlying pulp tissue (Yoshiba et al. 1996). They are positively immunostained for fibronectin and still remain positive a few days after capping, with corkscrew fiber-like structures visible between the cell bodies (Yoshiba et al. 1996). After $\mathrm{Ca}(\mathrm{OH})_{2}$ implantation for longer periods of time, $89 \%$ of the dentin bridges display tunnel defects, failing to provide a hermetic seal against infection to the underlying pulp. Due to microleakage, after 6 months, most of the $\mathrm{Ca}(\mathrm{OH})_{2}$ capping material disintegrates and disappears (Cox et al. 1996).

Similar molecular events have been identified when other $\mathrm{Ca}(\mathrm{OH})_{2}$ capping agents were used (Simon et al. 2008). After mild necrosis, the cells proliferate. At day 5 , cells implicated in an extracellular matrix formation express the cytoskeletal intermediary filament nestin. Just beneath the necrotic area, the cells are immuno-positive for osteopontin (OPN). Of note, OPN is implicated both in the control of inflammation and triggers the initiation of the pulp reparative process (Kuratate et al. 2008). Dentin matrix protein-1 (DMP-1) is one of the dentin noncollagenous phosphorylated extracellular matrix proteins (SIBLINGs) involved in the regulation of mineralization. DMP-1 induces the cytodifferentiation of dental pulp stem cells, becoming odontoblast-like cells (Lourenço Neto et al. 2015; Almushayt et al. 2006).

Capping with $\mathrm{Ca}(\mathrm{OH})_{2}$ is apparently the most efficient material utilized in direct reparative dentin formation (Decup et al. 2000; Andelin et al. 2003). Implantation with $\mathrm{Ca}(\mathrm{OH})_{2}$ revealed positive immunostaining for tenascin (TN) and fibronectin (FN). The reparative dentinal bridge was also immunostained for bone sialoprotein (BSP). After capping the pulp of the first maxillary molar, the pulp exposure is occluded by a bridge formed in 30 days (Decup et al. 2000; Andelin et al. 2003).

Hydroxide-containing pulp capping agent is widely used by dental practitioners, and Dycal appears to be the capping material most frequently employed by clinicians. In addition to the available $\mathrm{Ca}(\mathrm{OH})_{2}$ liners, recent studies shed lights on the possibility that self-assembling peptide hydrogels may contribute to support the differentiation of dental pulp stem cells ( $\mathrm{Li}$ et al. 2014). As new tools for tissue engineering, hydrogels constitute injectable scaffolds, and cell and bioactive molecule carriers (Cavalcanti et al. 2013; Goldberg et al. 2015).

A novel protein hydrogel forming a biodegradable cavity liner (in this report named Hydrogel) was developed, which has the potential to be used as a pulp capping agent. The chemical constituents of Hydrogel are purified protein, the bovine serum albumin (BSA), and glutaraldehyde (GA), a cross-linking agent. When activated by mixing and dispensed to target tissue sites, GA covalently cross-links the surface-proteins of the patient's tissue to BSA. The US Patent Application indicates the composition of BSA/GA, and it approves its sealing and regenerative features for tooth pulp. This material constitutes a resorbing and curable wound dressing and/or a biodegradable adhesive in dental restorative and endodontic procedures (Angeletakis 2014). It also contributes to the formation of a barrier against acidic monomers, namely to the etching agents used in dentistry. However, the mechanisms sustaining how hydrogels are acting and their molecular targets have not yet been elucidated.

The aim of this in vivo investigation was to compare the effects of Hydrogel, a bovine serum albumin (BSA)/ glutaraldehyde, and Dycal, a calcium hydroxide cavity liner used as pulp capping materials, on reparative and reactionary dentin formation in rat dental pulps following pulp exposure and capping. 


\section{Methods}

Animal and tooth preparation: reparative dentin formation after pulp capping

All animal experiments were performed under an institutionally approved protocol for the use of animals in research. Twenty-four 6-week-old Sprague-Dawley male rats (150 $\mathrm{g}$ at the beginning of the experiments) were obtained from the Janvier Laboratories (Le Genest-StIsle, France). They received drinking water ad libitum and were fed with specific food pellets during the tested time periods (first week, second week, third week, and fourth week). In each tested period, randomly selected 6 rats were sacrificed. They were anaesthetized intraperitoneally with $20 \%$ ketamine (Imalgene1000, Merial, France) and $5 \%$ xylazine (Rompun, Alcyon, France) before tooth preparation.

\section{Preparation of cavities, capping procedure}

The cavities were drilled within a few seconds under microscope, and the carbide tungsten burrs were water- cooled during the preparation in the occlusal aspect of the first maxillary molars (size of the bur: $0.6 \mathrm{~mm}, 0.05$ ISO). Random cavities were prepared in the central part of the teeth, to get good access to the enamel-dentin junction. The deepest part of the dentin cavity was pushed with a steel probe, producing a pulp exposure as previously described (Cox et al. 1996; Almushayt et al. 2006). This location maintains intact the resin filling during the period of time selected and prevents bacterial contamination from the oral cavity.

The exposed pulps were capped either with Hydrogel (Dentsply International Inc., York, PA, USA) (group 1G1, right molar) or with Dycal (Radiopaque Calcium Hydroxide Lining Material; Dentsply International) (group 2-G2, left molar). Pulp capping materials were prepared according to manufacturers' instructions. Base and catalyst pastes of Dycal were mixed in 1:1 proportions, while BSA:GA components of Hydrogel were mixed in 4:1 proportions. After setting of capping materials (Dycal set in 2-3 min, while Hydrogel set in 20-
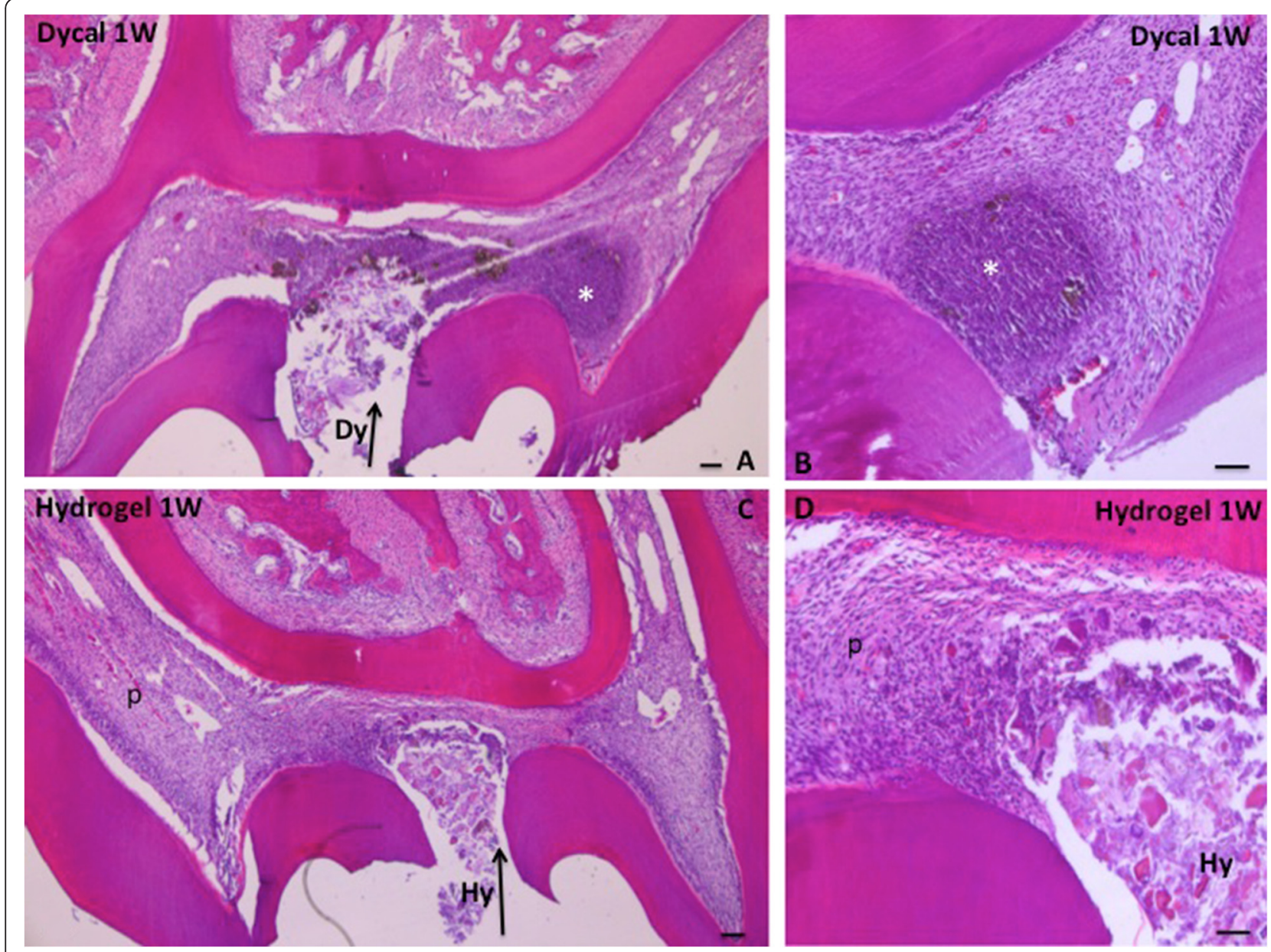

Fig. 1 a One week after Dycal capping, inflammation is seen in the dental pulp (asterisk). b Inflammation process 1 week after Dycal capping. c After Hydrogel capping for 1 week (Hy-arrow) pulp inflammation is discrete or lacking. $\mathbf{d}$ Pulp inflammation is barely detectable in the pulp. $P$ pulp, Bars: a, b $50 \mu \mathrm{m} ; \mathbf{c}$, d $100 \mu \mathrm{m}$ 
30 s) cavities were bonded (Prime \& Bond Elect Universal Adhesive; DENTSPLY Corporate York, USA) and sealed with SDR ${ }^{\mathrm{ma}}$ bulk-fill flowable composite (DENTSPLY Caulk, Milford, DE 19963).

\section{Histological procedures}

Under intraperitoneal anaesthesia, the 24 rats were perfused through the heart with a buffered $4 \%$ paraformaldehyde fixative solution injected with a Ministaltic pump. Bloc sections of the three maxillary molars were dissected out. The sections included the first molar and extended up to the third molar. They were fixed overnight and demineralized with $4.13 \%$ EDTA under microwave. The samples were dehydrated, embedded in wax, and sectioned (7- $\mu$ m-thick sections) (Decup et al. 2000).

After dewaxing, the sections were stained with hematoxylin-eosin and examined with a light microscope.
A series of staining methods were used, including Masson's trichrome, Mallory's staining, Alizarin red, and Alcian blue (at pH 2.3 in order to visualize glycosaminoglycans).

Measurements of the thickness of reactionary dentin were performed on figures printed at the same magnification with ImageJ software (National Institutes of Health, MD), 4 weeks after implantation of the capping agent. Despite the complexity of the tri-dimensional coronal pulp structure, the specific distribution of reparative dentin was evaluated after 1 month in Dycal- and Hydrogel-capped molars. Results were compared with Kruskal Wallis and Mann Whitney $U$ tests at $p=0.05$.

\section{Results}

One week after Dycal implantation in the dental pulp (Fig. 1a, b), a moderate inflammatory reaction (mild necrosis) was detectable at proximity of $\mathrm{Ca}(\mathrm{OH})_{2}$ used for pulp capping. Around pulpal cell

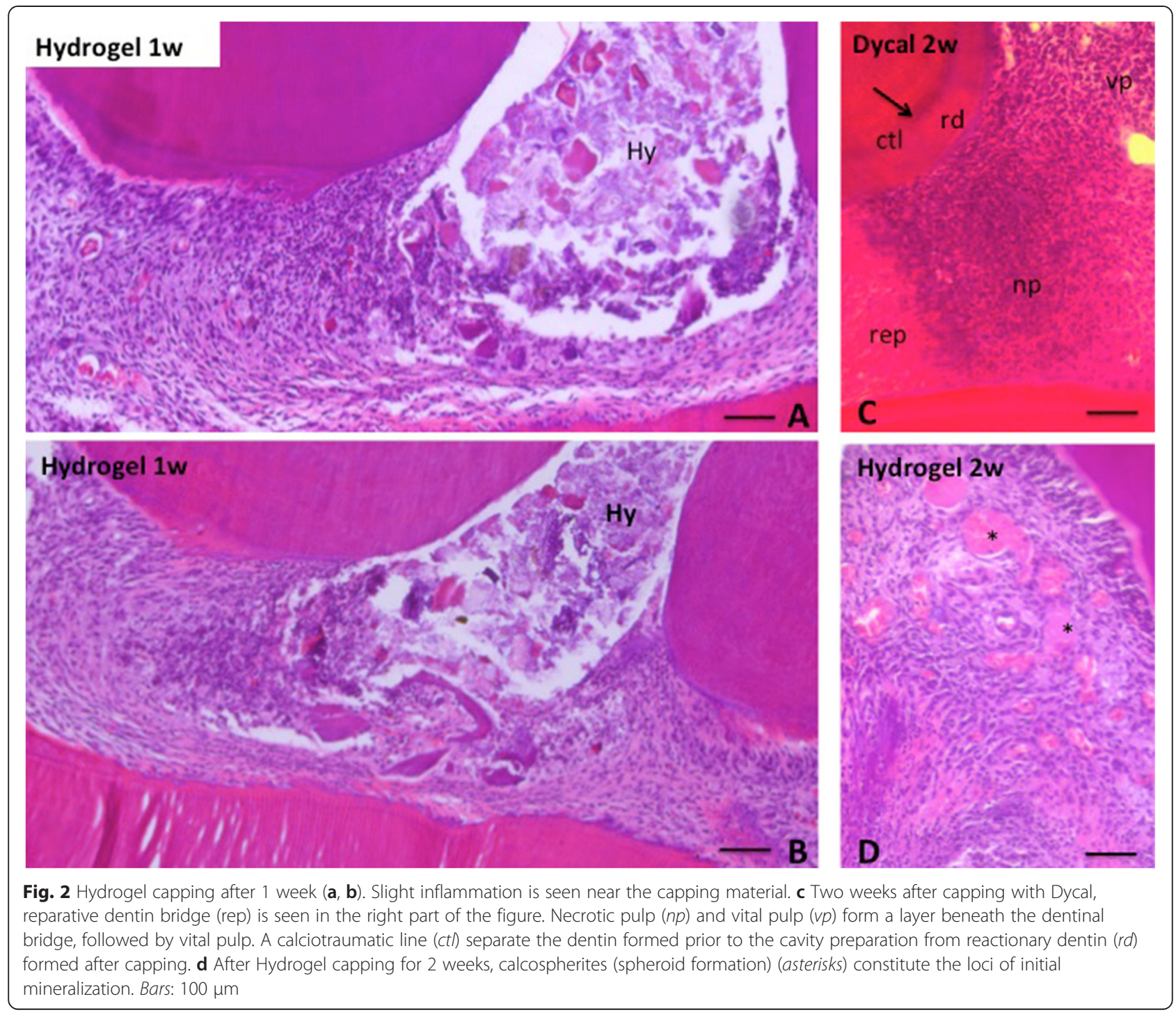


condensation, a half-moon layer contained a few expanded rows of cells. Just below the half-moon layer, no change was detectable within the pulp. One week after Hydrogel implantation (Figs. 1c, d and 2a, b), the inflammatory process was restricted to a small portion of the pulp.

After 2 weeks, an inflammatory process was seen in the Dycal capped pulp (Fig. 2c). Adjacent to reparative dentinal bridge (rep), a layer of necrotic pulp tissue (np) isolates this layer from the vital pulp (vp). Two weeks after Hydrogel capping (Fig. 2d), calcospheritic structures (spheroid formation) located at the periphery of the pulp initiate mineralization within the pulp (asterisks).

After 2 weeks, Alcian blue-positive areas (dotted arrow) $($ Fig. 3a-c) were present in the lateral regions adjacent to the Hydrogel $(\mathrm{Hy})$ and the same occurred after Dycal capping (Fig. 4a, b). The calciotraumatic lines were present in dentin (arrows) underlined the limits between the dentin already formed before the cavity preparation and the reactionary dentin after pulp exposure (Figs. 3a-c and $4 a, b$ ). Positively stained glycosaminoglycan-rich areas were present within the pulp $(\mathrm{AB}+)$, displaying the appearance of chondrocyte-like cells.

After pulp capping for 4 weeks, the pulp volume was largely reduced by a thick reactionary dentin layer (rd) but it also contained reparative dentin (rep), except in the non-mineralized mesial and distal areas (Fig. 5a, b). In molars implanted with Dycal for 4 weeks (Fig. 5a, b), the formation of reactionary dentin (rd) was enhanced, reducing the pulp volume (rep) (dark blue and brown). The thickness of reparative dentin varied between 100 and $200 \mu \mathrm{m}$, depending on the zone examined. The central and distal pulp horns contained unmineralized dentin (white asterisks). A reparative dentinal bridge was formed in the central portion of the pulp (arrows),

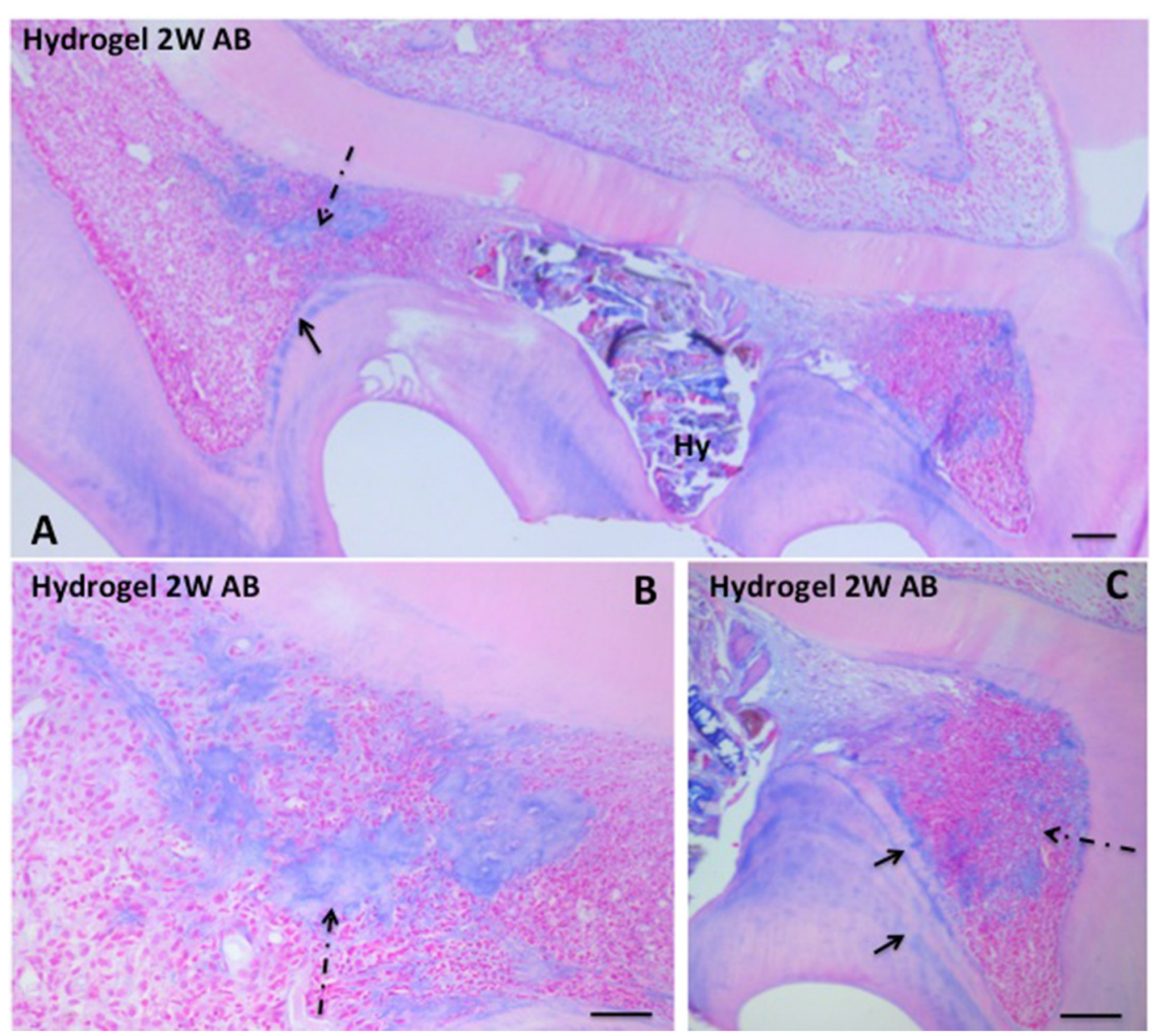

Fig. 3 a Alcian blue (AB)-positive staining 2 weeks after Hydrogel capping. Alcian blue structures are seen in the pulp (dotted arrow). Alcian bluepositive calciotraumatic lines (arrow) separate the dentin formed before the pulp exposure from the reactionary dentin formed after pulp capping. b After 2 weeks Hydrogel (Hy_dotted arrow) contributes to the formation of a reparative dentinal bridge. c After 2 weeks, Hydrogel capping induces two reactions: firstly calciotraumatic reactionary lines within dentin (arrows) and secondly Alcian blue-positive cell aggregates in the pulp (dotted arrow). Bars: $100 \mu \mathrm{m}$ 


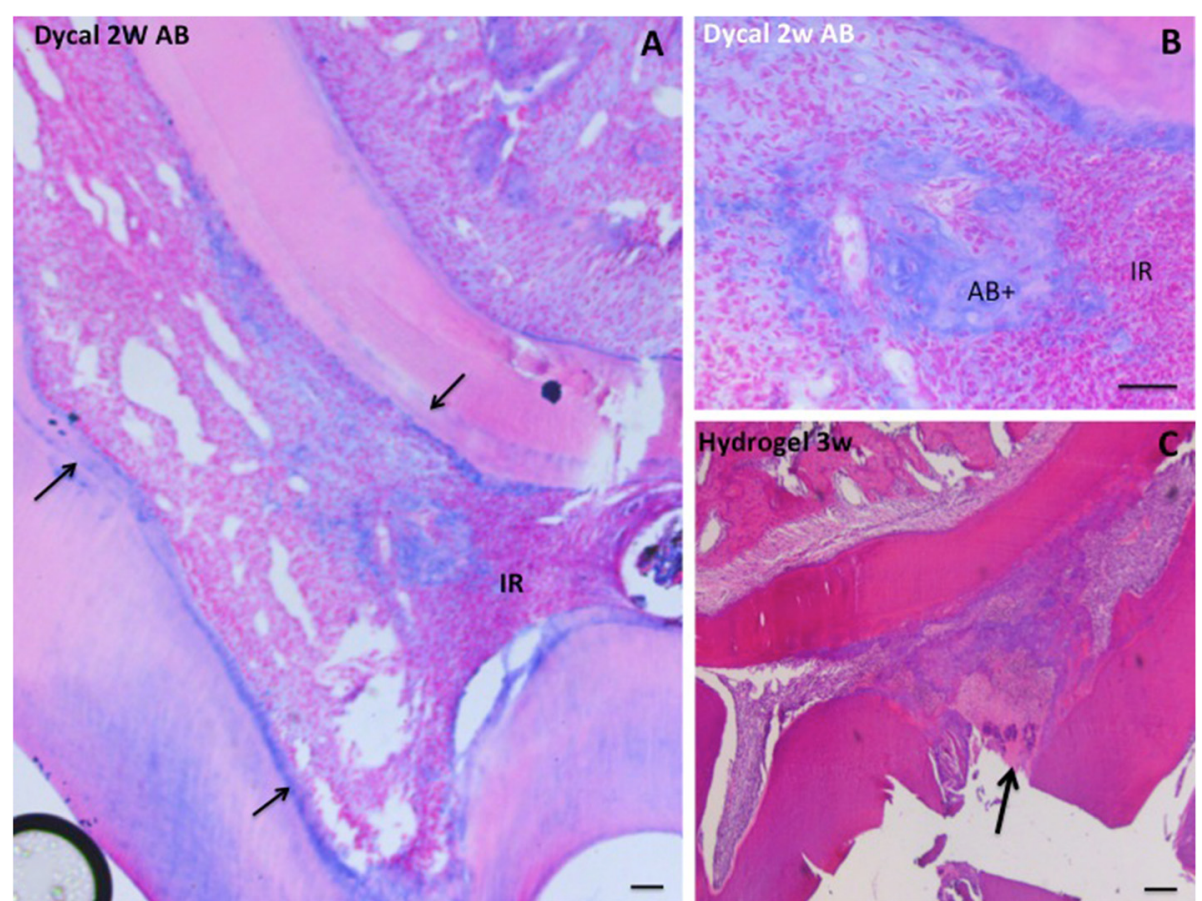

Fig. 4 a Dycal capping for 2 weeks. Black arrows indicate calciotraumatic lines. An Alcian blue aggregate is seen within the dental pulp. b The predentin is Alcian blue positive. Near the inflammatory reaction (IR), Alcian blue-positive reaction (AB+) located within the dental pulp may be a zone where pulp cell shifted to a chondrocyte phenotype. c Three weeks after pulp capping with Hydrogel, a reparative dentinal bridge (arrow) occludes the pulp exposure (arrow). Bars: $100 \mu \mathrm{m}$

displaying tunnel defects (black arrows) (Fig. 5a, b). In contrast, the mesial and distal portions of the pulp were not mineralized (white asterisks).

Four weeks after Hydrogel capping, the formation of reactionary dentin leads to the complete closure of the isthmus (arrows) (Fig. 6a). A large part of the mesial pulp was unmineralized (white asterisk), whereas in the distal horn, mineralized pulp horn were seen alone, proving a stronger mineral formation in G1 M. After Hydrogel capping, the thickness of reactionary dentin varied according to their location in the pulp chamber, and measurements indicated variations between 50 and $150 \mu \mathrm{m}$ (Fig. 6b-d). Isthmuses between the pulp horns were closed (Fig. 6a, arrows).

Figure $6 \mathrm{c}$ showed massive formation of reactionary (rd) and reparative dentin (rep), whereas the central part remained largely open depending on the size of the pulp exposure (Fig. 6d) (arrow). Inside each group of rats, the results were fairly homogenous, individual variations being mostly associated with the diameter of the pulp exposure. Most of the exposed pulp had a size identical to the bur that was used; however, a very few were larger (Fig. 6d).

\section{Discussion}

The aim of the present investigation was to discriminate pulp capping efficiency of Hydrogel and Dycal and evaluate their specificity emerging from their respective effects (Janebodin et al. 2010). Dycal is a calcium hydroxide cavity liner extensively used by dental practitioners. In contrast, MTA is employed mostly for root perforations and less for pulp capping (Bogen et al. 2008; Zhu et al. 2015). This is why we focus here essentially on the comparison between Hydrogel and Dycal.

Our results evidence that pulp capping with Dycal, as well as with Hydrogel, stimulates the formation of reactionary dentin at the periphery of the pulp chamber. Dycal contributes to the partial closure of the pulp exposure, producing a defective reparative dentinal bridge. Hydrogel contributes more efficiently to the construction of a reparative dentin, which looks homogeneous. The comparison between the two capping agents shed lights on the actual differences between Dycal capping, inducing an inflammatory process, closely associated with the presence of necrotic areas, and Hydrogel treatment where inflammatory pulp cells were undetectable. The role of inflammation in the repair process seems to be a crucial point (Goldberg et al. 2015).

According to the Patent Application Publication, Hydrogel is formed initially by $40-47$ wt.\% BSA, mixed with a solution of 5-15 wt.\% GA in a ratio of $4: 1$ by volume (Angeletakis 2014). Biochemical changes and cytotoxicity are associated with the degradation of polymeric GAderived cross-links (Huang-Lee et al. 1990). Collagen 

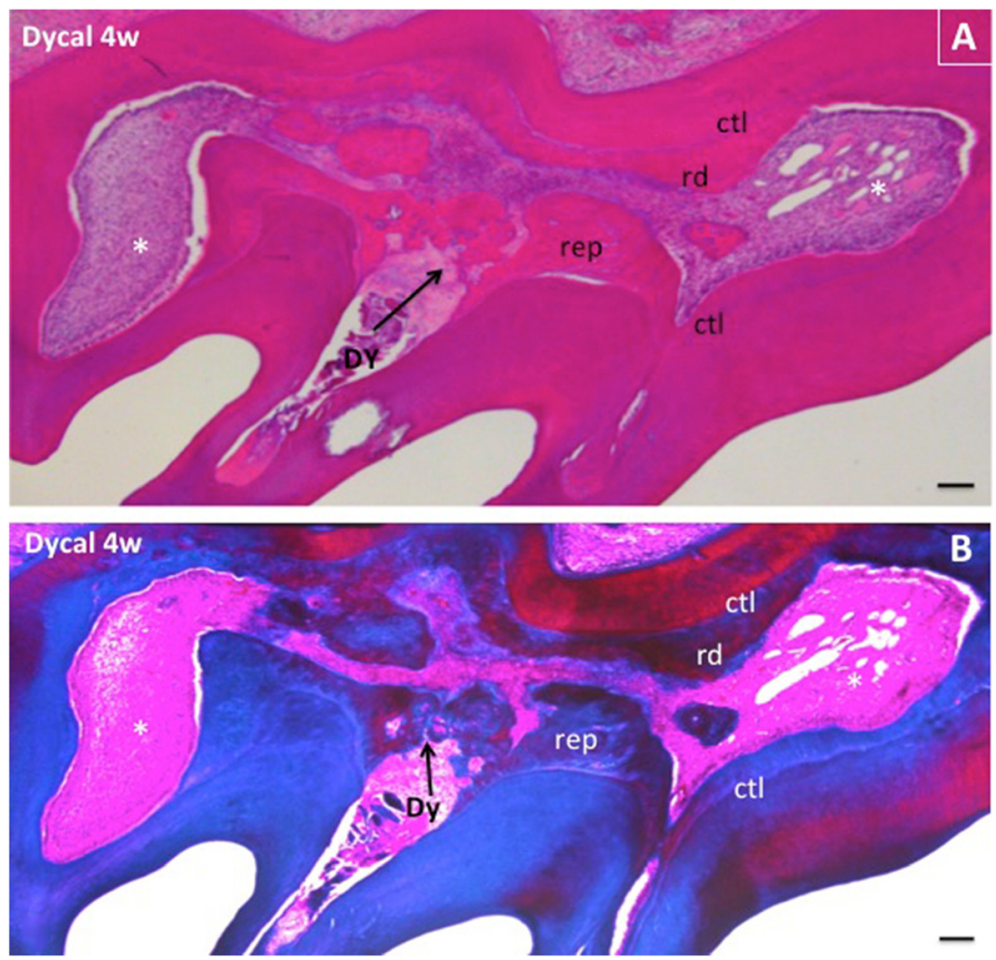

Fig. 5 a, b Formation of a reparative dentinal bridge (rep) 4 weeks after pulp capping (Alizarin red and Masson's trichrome). Tunnels crossing the reparative dentin formation prevent the total closure of the bridge. Reactionary dentin ( $r d$ ) is separated from the dentin layer formed prior capping by calciotraumatic lines (ct). The mesial and distal pulp horns remain non-mineralized (white asterisks). Bars: $100 \mu \mathrm{m}$

requires cross-linking by reagents such as GA, potentially cytotoxic. We assume that apoptosis and/or nemosis may be involved in the mechanism of GA toxicity of crosslinked collagen (Gough et al. 2002). Metalloprotease degradation of Hydrogel contributes to the releases of GA, also known to be a cell fixative. This release supports BSA bioactivity, associated with pulp repair. After Hydrogel capping inflammation was weak or barely detectable. By contrast, Dycal acting continuously as a highly alkaline compound produces a superficial burn covering a scar at the pulp surface, and consequently producing a limited area of pulp inflammation. Two weeks after Dycal capping, a mild necrotic area was observed at the limit between the reparative dentinal bridge and vital pulp. After 4 weeks, inflammatory processes were still obvious. Therefore, differences were clearly recognized between the two capping agents.

Round or globular structures (spheroid formation) forming clusters were noticeable in the Hydrogel-treated pulp. The nemosis-induced inflammatory reaction stimulates the dental pulp, leading to massive proinflammatory, proteolytic, and growth factor responses. The programmed cell death included the release of cycloxygenase-2, prostaglandin E2, interleukin-8, and several MMPs (Goldberg et al. 2015). The globular structures seen only after Hydrogel capping seem to be connected with the initial steps of mineralization. Later, calcospherites expanding in size invaded gradually the pulp, suggesting that Hydrogel display a close partnership with nemosis.

The Alcian blue-positive staining was identified along the calciotraumatic lines and within the dental pulp. This implies that in the regenerating dental pulp, cell aggregates display high glycosaminoglycan content, suggesting that some pulp cells acquire precociously a chondrocyte phenotype and afterward give rise to osteoblast-like cells. Pulp multipotent or totipotent cells differentiate into odontoblasts, osteoblasts, adipocytes, neural cells and chondrocytes, depending on the culture medium (Gough et al. 2002). Magloire et al. (1992) have previously shown that under a carious lesion, pulp cells elaborate cartilagelike aggregates identified by immunohistochemistry and their type II and XI collagen content. It is also well documented that the clonal cell line A4 contributes to dentin-like and/or bone formation. Pulp cells have the capacity to differentiate into neuronal cells, adipocytes and chondrocytes, whereas the C5 and H8 pulp clones displayed more restricted potentials (Lacerda-Pinheiro et al. 2012). Expression of type II collagen and aggrecan confirmed chondrogenic conversion (Huang-Lee et al. 1990; Iohara et al. 2006). After Hydrogel and/or Dycal capping, Alcian blue 

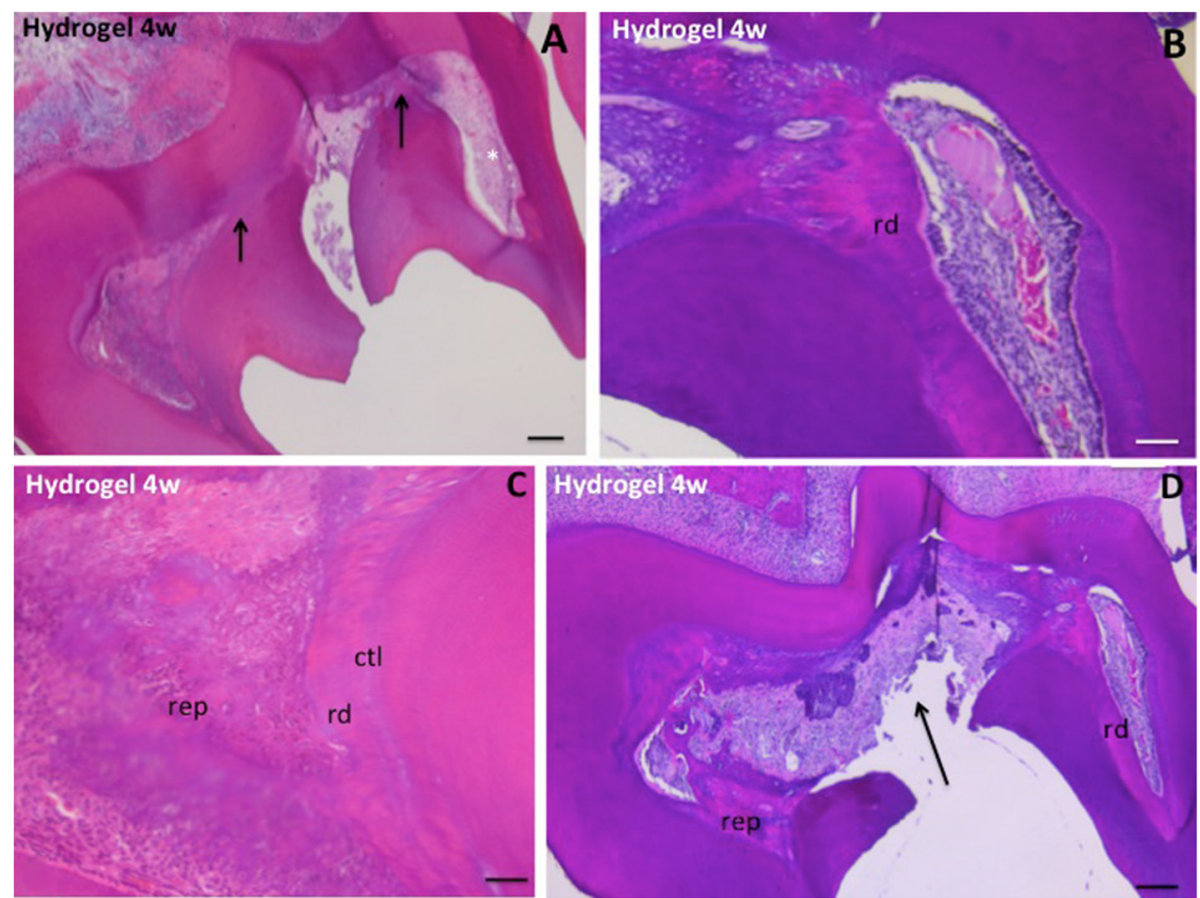

Fig. 6 a After 4 weeks of Hydrogel capping, the figure shows the closure of the isthmus located between the pulp horns (arrows). The mesial pulp horn is unmineralized (white asterisk). b Reactionary dentin ( $r d$ ) is massively formed 4 weeks after capping in the mesial part of the molar. $\mathbf{c}$ Reparative dentin (rep) fills partially the distal horn. Behind a calciotraumatic line (ct), reactionary dentin ( $r d$ ) is appended. $\mathbf{d}$ Large preparation (arrow) of the occlusal cavity cannot be repaired spontaneously. Reparative (rep) and reactionary ( $r d$ ) dentins are formed in the mesial and distal parts of the pulp. Bars: $100 \mu \mathrm{m}$

positivity is apparently independent from the healing/mineralizing processes and shows a variable distribution. GAGs and/or chondrocytes are identified within the regenerating dental pulp. The mixture of reactionary and reparative dentin detected after pulp capping does not allow associating the capping molecule and a specific functional role. Hydrogel allows a polymerization reaction based on the cross-linked proteinaceous material of animal origin, acting as a biodegradable cavity liner, while Dycal is based on chemical calcium hydroxide properties (Angeletakis 2014).

Within the period of time considered, Dycal capping contributes to the heterogeneous formation of a reparative dentinal bridge. Tunnel defects as well as the presence of osteodentin favor bacteria recontamination and consequently the lack of formation of a hermetic seal. Reparative dentin bridge contains cell debris permitting communication between the oral cavity and the outer pulp. This is probably the reason for the long-term high rate failure of pulp cappings (Cox et al. 1996).

Using the rat model, the present study demonstrates some limitations. Rats do not necessarily provide an ideal model for pulp regeneration studies, and very different effects may be found in human. However, such an experimental approach allows easy assumptions.

\section{Conclusions}

To conclude, the formation of reactionary and reparative dentin was enhanced by Hydrogel capping compared to Dycal capping. The isthmus located between the pulp horns collapsed and merged. Three weeks after Hydrogel capping, the dentinal bridge occluding the pulp exposure was homogeneous, in contrast with the conclusions previously reported obtained after Dycal capping. Therefore, substantial differences were evident between a solid Hydrogel-induced dentinal bridge and tunnel disturbances shown after Dycal capping. Hence, Hydrogel might present some clinical advantages over Dycal capping.

\section{Competing interests}

The authors declare that they have no competing interests.

\section{Authors' contributions}

$\mathrm{AN}, \mathrm{EU}$, and $\mathrm{HAO}$ are in charge of animal experiments and preparing and staining tissue sections for microscopic evaluation; SS is in charge of opening cavities, pulp capping and composite fillings; $A B$ and OK are head of the labs, in charge of language corrections, editing of manuscript; MG is in charge of the evaluation of tissue sections regarding dentin formation, inflammation, etc. All the coauthors did substantial contributions to the conception, analysis of data, and interpretation All authors read and approved the final manuscript.

\section{Acknowledgements}

The Dentsply Company provided the capping agents and restorative materials. The not-commercially available Hydrogel was a gift from Dentsply (http://www.google.com/patents/US20140227665). 
The authors declare no potential conflicts of interest with respect to the authorship and/or publication of this article.

\section{Author details}

${ }^{1}$ Faculté des Sciences Fondamentales et Biomédicales, INSERM UMR-S 1124 and Université Sorbonne Paris Cité, 45 rue des Saints Pères, 75270 Cedex 06 Paris, France. ${ }^{2}$ INSERM UMRS 1138 Université Paris Diderot, SPC, Université Pierre et Marie Curie, 15 rue de l'Ecole de Médecine, 75006 Paris, France.

Received: 23 December 2015 Accepted: 30 March 2016

Published online: 03 May 2016

\section{References}

Almushayt A, Narayanan K, Zaki AE, George A. Dentin matrix protein 1 induces cytodifferentiation of dental pulp stem cells into odontoblasts. Gene Ther. 2006;13:611-20

Andelin WE, Shabahang S, Wright K, Torabinejad M. Identification of hard tissue after experimental pulp capping using dentin sialoprotein (DSP) as a marker. J Endod. 2003;29:646-50.

Angeletakis C. Resorbable and curable compositions for use in dentistry. Patent Application Publication. USA. 2014. US 2014/0227665 A1.

Bogen G, Kim JS, Bakland LK. Direct pulp capping with mineral trioxide aggregates - an observational study. JADA. 2008;139:305-15.

Cavalcanti BN, Zeitlin BD, Nor JE. A hydrogel scaffold that maintains viability and supports differentiation of dental pulp stem cells. Dent Mater. 2013:29:97-102.

Cox CF, Subay RK, Ostro E, Suzuki S, Suzuki SH. Tunnel defects in dentin bridges: their formation following direct pulp capping. Oper Dent. 1996;21:4-11.

Decup F, Six N, Palmier B, Buch D, Lasfargues JJ, Salih E, et al. Bone sialoprotein-induced reparative dentinogenesis in the pulp of rat's molar. Clin Oral Investig. 2000;4:110-9.

Goldberg M, Njeh A, Uzunoglu E. Is pulp inflammation a prerequisite for pulp healing and regeneration? Med Inflamm 2015; doi:10.1155/2015/347649.

Gough JE, Scotchford CA, Downes S. Cytotoxicity of glutaraldehyde crosslinked collagen/poly (vinyl alcohol) films is by the mechanism of apoptosis. J Biomed Mater Res Part A. 2002;61:121-30.

Hermann BW. Dentinobliteration der Wurzelkanäle nach Behandlung mit Calcium. Zahnarztl Rundsch. 1930;39:888-99.

Huang-Lee LLH, Cheung DT, Nimni M. Biochemical changes and cytotoxicity associated with the degradation of polymeric glutaraldehyde derived crosslinks. J Biomed Mater Res Part A. 1990;24:1185-201.

lohara K, Zheng L, Ito M, Tomokiyo A, Matsushita K, Nakashima M. Side population cells isolated from porcine dental pulp tissue with self-renewal and multipotency for dentinogenesis, chondrogenesis, adipogenesis, and neurogenesis. Stem Cells. 2006;24:2493-503.

Janebodin K, Horst OV, Osathanon T. Dental pulp responses to pulp capping materials and bioactive molecules. CU Dent J. 2010;33:229-48.

Kuratate M, Yoshiba K, Shigetani Y, Yoshiba N, Ohshima H, Okiji T. Immunohistochemical analysis of nestin, osteopontin, and proliferating cells in the reparative process of exposed dental pulp capped with mineral trioxide aggregate. J Endod. 2008;34:970-4.

Lacerda-Pinheiro S, Dimitrova-Nakov S, Harichane Y, Souyri M, Petit-Cocault L, Legrès $L$, et al. Concomitant multipotent and unipotent dental pulp progenitors and their respective contribution to mineralised tissue formation. Eur Cell Mater. 2012;23:371-86

Li F, Liu X, Zhao S, Wu H, Xu HH. Porous chitosan bilayer membrane containing TGF-beta1 loaded microspheres for pulp capping and reparative dentin formation in a dog model. Dent Mater. 2014;30:172-81.

Lourenço Neto N, Marques NC, Fernandes AP, Rodini CO, Sakai VT, Abdo RC, et al. Immunolocalization of dentin matrix protein-1 in human primary teeth treated with different pulp capping materials. J Biomed Mater Res B Appl Biomater 2015. doi: 10.1002/jbm.b.33379. [Epub ahead of print].

Magloire $H$, Bouvier $M$, Joffre A. Odontoblast response under carious lesions. Proc Finn Dent Soc. 1992;88 Suppl 1:257-74.

Schroder U. Effects of calcium hydroxide-containing pulp-capping agents on pulp cell migration, proliferation, and differentiation. J Dent Res. 1985;64:541-8.

Simon S, Cooper P, Smith A, Picard B, Ifi CN, Berdal A. Evaluation of a new laboratory model for pulp healing: preliminary study. Int Endod J. 2008;41:781-90.

Tronstad L. Reaction of the exposed pulp to Dycal treatment. Oral Surg Oral Med Pathol. 1974;38:945-53.
Yoshiba K, Yoshiba N, Nakamura H, Iwaku M, Ozawa H. Immunolocalization of fibronectin during reparative dentinogenesis in human teeth after pulp capping with calcium hydroxide. J Dent Res. 1996;75:1590-7.

Zhu C, Ju B, Ni R. Clinical outcome of direct pulp capping with MTA or calcium hydroxide: a systematic review and meta-analysis. Int J Clin Exp Med. 2015:8:17055-60.

\section{Submit your manuscript to a SpringerOpen ${ }^{\circ}$ journal and benefit from:}

- Convenient online submission

- Rigorous peer review

- Immediate publication on acceptance

- Open access: articles freely available online

- High visibility within the field

- Retaining the copyright to your article

Submit your next manuscript at springeropen.com 